\section{Los linfocitos B y las inmunodeficiencias primarias}

Gabriela López-Herrera

\section{Resumen}

Las inmunodeficiencias primarias de anticuerpos representan las enfermedades genéticas del sistema inmunitario más frecuentes y las primeras en ser reconocidas durante la historia de la inmunología. Los anticuerpos se reconocieron como parte de la respuesta inmunitaria humoral desde hace más de un siglo, tiempo después de su descubrimiento se reconoció la primera inmunodeficiencia primaria de anticuerpos: la agammaglobulinemia, seguida por la inmunodeficiencia común variable y el síndrome de hiper-lgM. Los descubrimientos subsecuentes en la historia de la inmunología facilitaron el entendimiento de la patología de estas enfermedades; por ejemplo, el descubrimiento de los linfocitos $B$, de las células B inmaduras en médula ósea, de la señalización del receptor de antígeno en estas células, entre muchos otros mecanismos celulares y moleculares. Las inmunodeficiencias humorales se han estudiado ampliamente y también han apoyado los avances científicos para la comprensión de los mecanismos inmunológicos que tienen lugar en nuestro organismo. Esta revisión documental pretende revisar los hallazgos relevantes en la historia del linfocito B y su conexión con el descubrimiento de nuevas inmunodeficiencias primarias de anticuerpos con el objetivo de mostrar que la generación del conocimiento científico tiene una aplicación directa en el entendimiento de los mecanismos moleculares que se ven afectados en este tipo de defectos.

PALABRAS CLAVE: inmunodeficiencias primarias de anticuerpos, enfermedades genéticas del sistema inmunitario, agammaglobulinemia, linfocitos B.

\section{Lymphocytes B and primary immunodeficiencies.}

Gabriela López-Herrera

\begin{abstract}
Primary antibody deficiencies represent the most frequent genetic diseases of the immune system and the first to be recognized along immunology history. The antibodies were recognized as part of the humoral immune system long ago, and after immunoglobulin discovery, the first antibody immunodeficiency were recognized and named as "agammaglobulinemia", followed by the common variable immuno-
\end{abstract}

Unidad de Investigación en Inmunodeficiencias, Instituto Nacional de Pediatría, Secretaría de Salud, Ciudad de México.

Recibido: 22 de septiembre 2015

Aceptado: 6 de enero 2016

Correspondencia

Dra. Gabriela López Herrera

lohegabyqbp@gmail.com

Este artículo debe citarse como

López-Herrera G. Los linfocitos B y las inmunodeficiencias primarias. Rev Alerg Méx. 2016 enemar;63(1):58-70. 
deficiency and the hyper-IgM syndrome. The following discoveries in immunology history made possible the understanding of these pathologies, for example: the discoveries of B cells, pre-B cells, the signaling pathway directed by the antigen receptor and many other cellular and molecular mechanisms. Primary antibody deficiencies have been studied for a long time and the discoveries of new syndromes have been helpful in the understanding of immunological mechanisms that take place in our organism. Then, this manuscript pretends to review the relevant findings in the history of immunology, focused on the $B$ cells and the connection with the description of representative clinical entities of primary antibody deficiencies. The aim of this manuscript is to show to the reader that the generation of scientific knowledge has a direct application in the understanding of the molecular mechanisms that are affected in these diseases.

KEYWORDS: primary antibody deficiencies; genetic diseases of the immune system; agammaglobulinemia; B cells
Unidad de Investigación en Inmunodeficiencias, Instituto Nacional de Pediatría, Secretaría de Salud, Ciudad de México.

\section{Correspondence}

Dra. Gabriela López Herrera lohegabyqbp@gmail.com

\section{Inmunodeficiencias primarias}

Las inmunodeficiencias primarias son enfermedades que se distinguen por alteraciones genéticas que afectan la diferenciación o la función de las células del sistema inmunitario, lo que conlleva a una susceptibilidad exacerbada a contraer infecciones graves por diferentes tipos de microorganismos. Las inmunodeficiencias primarias son resultado de mutaciones en genes que codifican para proteínas importantes para la diferenciación de las células del sistema inmunitario o, bien, proteínas que participan en vías de señalización útiles para efectuar su función (producción de anticuerpos, citocinas, especies reactivas de oxígeno, etc.). ${ }^{1}$

Las inmunodeficiencias primarias se clasifican en diversos grupos de acuerdo con el componente del sistema inmunitario que se ve afectado, así tenemos, por ejemplo, inmunodeficiencias primarias en las que se ve afectada la función de los fagocitos, los linfocitos T, el complemento y de manera importante, la producción de anticuerpos o inmunoglobulinas. Las inmuno- deficiencias primarias de anticuerpos (también conocidas como humorales) son los trastornos diagnosticados con más frecuencia en todo el mundo; aproximadamente $55 \%$ del total de las inmunodeficiencias primarias diagnosticadas son de este tipo (http://esid.org/Working-Parties/ Registry/ESID-Database-Statistics).

Las inmunodeficiencias primarias humorales, a su vez, se subclasifican en tres tipos principales: la agammaglobulinemia (caracterizada por la disminución drástica de todos los isotipos de inmunoglobulinas), la inmunodeficiencia común variable (en la que existe una reducción de las concentraciones de dos de los tres isotipos de inmunoglobulinas que se encuentran en suero: $\lg \mathrm{M}, \lg \mathrm{G}$ e $\lg \mathrm{A}$ ) y el síndrome de hiper-IgM, en el que sólo hay producción de IgM y la producción de IgG e IgA está disminuida; ${ }^{2}$ existe, además, una serie de defectos humorales cuyas causas en la mayoría de los pacientes aún se desconocen, como la deficiencia de IgA, la hipogammaglobulinemia transitoria de la infancia, la deficiencia de subclases de IgG y la deficiencia específica de anticuerpos. El descubrimiento de estas en- 
fermedades está estrechamente asociado con el descubrimiento del linfocito B; los aspectos históricos que ligan estas enfermedades con la función y la diferenciación del linfocito B se detallan a lo largo de esta revisión.

\section{Aspectos históricos de las inmunodeficiencias de células $B$}

Uno de los reportes más antiguos que pueden encontrarse de deficiencias inmunológicas es un reporte publicado por Glanzmann y Riniker, en 1950, quienes comunicaron un caso clínico conformado por un síndrome de "agammaglobulinemia y linfocitoptisis" que después se llamó "agammaglobulinemia tipo suizo"; los pacientes con este síndrome se caracterizaban por la aparición temprana de los síntomas y la fatalidad de la misma. A pesar de que Glanzman y Riniker fueron los primeros en reportar el fenotipo clínico de la inmunodeficiencia combinada severa, no pudieron reconocerla como tal, pues reportaron que ese fenotipo se debía a infecciones severas por Candida albicans. ${ }^{3}$

Además, desde hace más de medio siglo, tras el descubrimiento de las anticuerpos, se ha descrito una serie de síndromes clínicos de causa genética que afectan al sistema inmunológico. El ejemplo más característico de esto es la agammaglobulinemia ligada al cromosoma X (XLA), descrita en 1952 por Ogden C Bruton, quien reportó en ese entonces el seguimiento clínico y de laboratorio de un paciente pediátrico que desde edades tempranas había sufrido diversos cuadros infecciosos causados por Streptococcus pneumoniae, casi siempre de la misma cepa; en esa época ya era conocido el fenómeno de memoria inmunológica, dada la protección frente a diversas infecciones que se conseguía con la vacunación; ${ }^{4}$ a este respecto, el paciente reportado por Bruton parecía carecer de memoria inmunológica. En ese mismo artículo, Bruton determinó una carencia de inmunoglobulinas en el suero de este paciente $y$, tras administrar de manera pasiva anticuerpos de individuos sanos, observó mejoría clínica en él. ${ }^{5}$ Las observaciones realizadas por Bruton lo llevaron a reunirse con Charles A Janeway (en el Boston Children's Hospital), ambos examinaron y comunicaron una serie de cinco casos con un curso clínico similar en la reunión de la Sociedad de Investigación Pediátrica (PRS), en Atlantic City, en 1952; sin embargo, Janeway nunca publicó los cuatro casos observados en Boston.?

Uno de los cinco pacientes reportados por Bruton y Janeway en el congreso de la Sociedad de Investigación Pediátrica tomó relevancia en años posteriores, porque, a pesar del tratamiento con gammaglobulina humana, este paciente tuvo complicaciones graves, fue hospitalizado por hematuria debido a una glomerulonefritis posestreptocócica. Además, Fred Rosen y Charles A Janeway reportaron la existencia de concentraciones elevadas en suero de anticuerpos 19S (IgM) y disminuidas de anticuerpos 7S (IgG), identificando el síndrome que ahora se conoce como síndrome de hiper-lgM (HIGM). ${ }^{8}$ Durante mucho tiempo este síndrome se consideró un defecto intrínseco de las células B; sin embargo, estudios posteriores demostraron que el defecto funcional estaba en los linfocitos T; en este caso, de un tumor de linfocitos T (síndrome de Sézary) que inducía de manera activa la recombinación del cambio de isotipo, se utilizó para inducir cambio de isotipo en células $B$ de pacientes con síndrome de hiper-lgM. ${ }^{9}$ Sin embargo, existen diferentes variedades del síndrome de hiper-lgM y varios de éstos muestran, efectivamente, defectos intrínsecos de la célula B.

Charles Janeway acuñó el término de inmunodeficiencia común variable para englobar a los pacientes con concentraciones disminuidas de inmunoglobulinas (pero no ausentes); también reportó la forma transitoria de la agammaglobulinemia, ahora nombrada hipo- 
gammaglobulinemia transitoria de la infancia; ${ }^{10,11}$ más tarde, estos términos los retomó Max D Cooper, quien en 1978 publicó la primera clasificación de las inmunodeficiencias primarias. ${ }^{12}$

La deficiencia de $\operatorname{IgA}(\operatorname{IgAd})$, una de las inmunodeficiencias primarias humorales más frecuentes, se describió en 1966 en pacientes adultos con diarrea, absorción intestinal reducida y con episodios de bronconeumonía; ${ }^{13}$ seguido a este hallazgo, se describió la deficiencia de subclases de IgG, en 1970, por un grupo liderado por Fred Rosen, quienes determinaron esta inmunodeficiencia en pacientes que padecían infecciones de repetición por microorganismos piógenos; ${ }^{14}$ la deficiencia selectiva de anticuerpos frente a polisacáridos bacterianos se describió en 1985, cuando Raif S Geha reportó una serie de pacientes con infecciones sinopulmonares recurrentes y con títulos disminuidos de anticuerpos contra polisacáridos de Haemophilus influenzae tipo B. ${ }^{15}$

En la Figura 1 se muestran algunos descubrimientos respecto a la biología, desarrollo y función del linfocito B y el descubrimiento de las principales inmunodeficiencias primarias de anticuerpos que se estudian en la actualidad.

\section{Frecuencia de las inmunodeficiencias} humorales

En la actualidad, existen comunidades científicas que se dan a la tarea de registrar y organizar una base de datos que contiene la información acerca de los tipos de diagnóstico de inmunodeficiencias primarias que se realizan. La Sociedad Europea de Inmunodeficiencias (ESID) es, quizá, la comunidad científica que ha presentado datos relevantes al respecto. En la página electrónica de esa sociedad, http://esid.org/Working-Parties/ Registry/ESID-Database-Statistics puede notarse que las inmunodeficiencias primarias de anticuerpos se han considerado los defectos más comúnmente diagnosticados en el mun- do y abarcan en la actualidad cerca de $60 \%$ de los diagnósticos. Los principales grupos de inmunodeficiencias primarias que afectan la diferenciación y la función de las células B son: la agammaglobulinemia, el síndrome de hiperIgM y la inmunodeficiencia común variable. Sin embargo, existen muchos otros defectos en los que la célula B se ve afectada en su función o diferenciación; sin embargo, estos defectos forman parte de otro tipo de inmunodeficiencias primarias, como los defectos combinados de células $\mathrm{T}$ y $\mathrm{B}$, síndromes complejos como ataxia-telangiectasia o síndrome de Griscelli, entre otros.

La IgAd es la inmunodeficiencia primaria más frecuente, su prevalencia es de 1/500 en población caucásica, ${ }^{16}$ mientras que la hipogammaglobulinemia transitoria de la infancia, la deficiencia de subclases de IgG y la deficiencia selectiva de anticuerpos son enfermedades raras y no existen estadísticas confiables respecto a su ocurrencia. Además, los mecanismos inmunológicos asociados con estas inmunodeficiencias se han estudiado poco, por lo que en este artículo abordaremos las tres inmunodeficiencias primarias humorales mayormente estudiadas a lo largo de la historia de la inmunología: la agammaglobulinemia, el síndrome de hiper-IgM y la inmunodeficiencia común variable.

\section{Determinación de las deficiencias genéticas en} las inmunodeficiencias primarias

Para el decenio de 1960 ya era clara la existencia de las inmunodeficiencias primarias; sin embargo, no se conocía el defecto genético que originaba ninguno de los síndromes descritos en esa época. En 1972 y 1975, Eloise Giblett determinó dos defectos genéticos en pacientes con inmunodeficiencia combinada severa (SCID); el primero de ellos, la deficiencia de adenosina desaminasa (ADA), se encontró de manera 


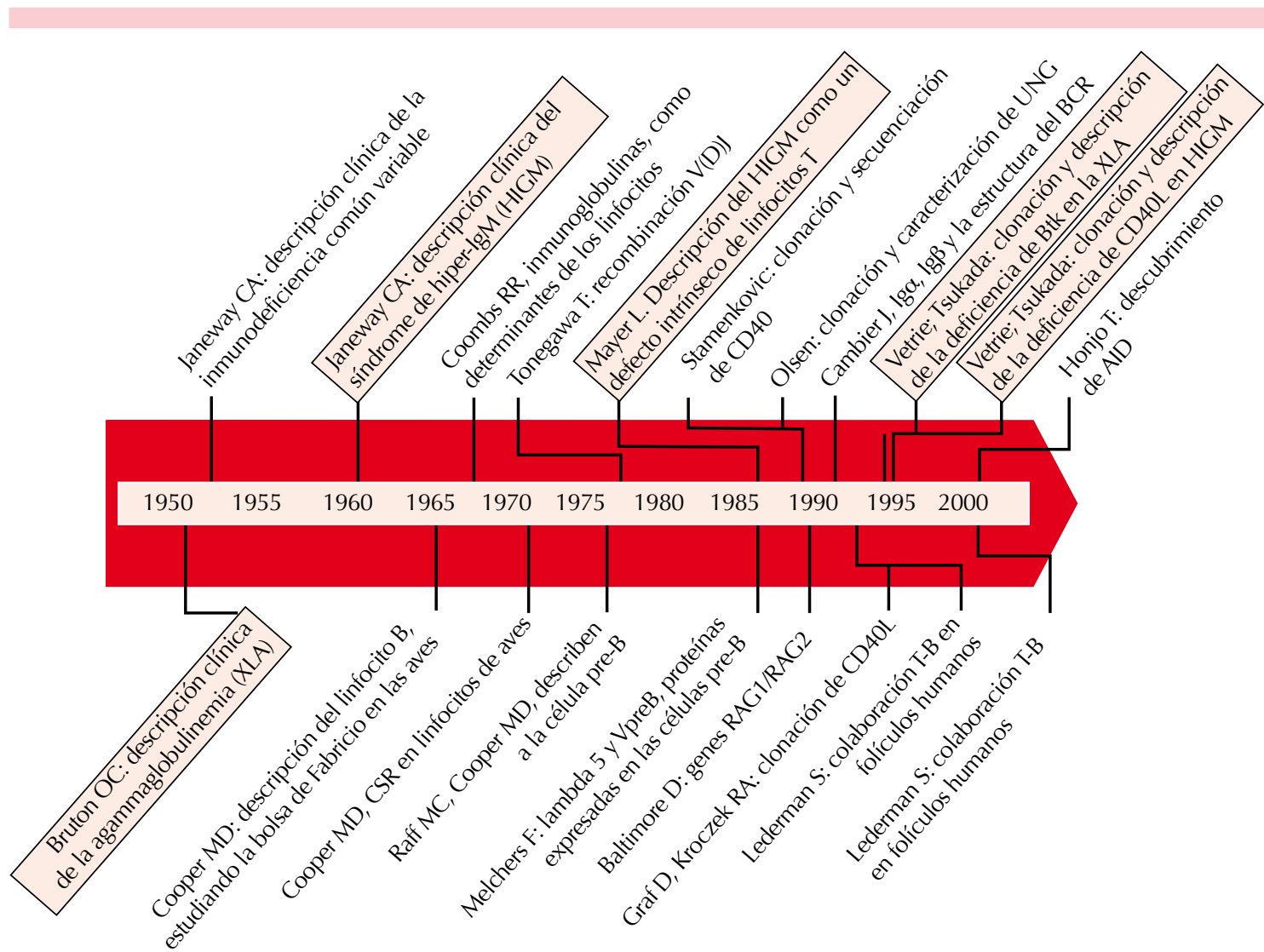

Figura 1. Descubrimientos respecto a la biología, desarrollo y función del linfocito B y el descubrimiento de las principales inmunodeficiencias primarias de anticuerpos que se estudian en la actualidad.

fortuita cuando Hillary Mewissen contactó a Eloise Giblett con el objetivo de determinar algunos marcadores polimórficos en un paciente con inmunodeficiencia combinada severa para realizarle un trasplante; uno de estos marcadores polimórficos que se utilizaban en el laboratorio de Eloise Giblett era precisamente adenosina desaminasa; este paciente carecía de la actividad de esta enzima. ${ }^{17}$ Los avances tecnológicos, como los métodos de secuenciación del ADN y la reacción en cadena de la polimerasa (PCR) por Frederick Sanger y Kary Mullins, respectivamente, ${ }^{7}$ dieron la pauta para determinar muchos de los defectos genéticos asociados con las inmunodeficiencias primarias y la actual tecnología de secuenciación masiva del ADN ha resultado en que se reporten con una frecuencia aún mayor nuevos defectos genéticos asociados con estas enfermedades.

\section{Agammaglobulinemia}

Los avances en el conocimiento de mecanismos inmunológicos también facilitaron la búsqueda de estos defectos genéticos; por ejemplo, el descubrimiento de las células pre-B ocurrió a mediados del decenio de 1970;18 la descripción de los componentes del receptor de la célula preB (pre-BCR) se realizó en los decenios de 1980 y $1990^{19,20}$ y la descripción de uno de los com- 
ponentes de la vía de señalización del pre-BCR se describió gracias a la agammaglobulinemia ligada al cromosoma $X$; dos grupos independientes ${ }^{21,22}$ mapearon, clonaron y describieron la secuencia codificante de la ahora conocida tirosina cinasa de Bruton (Btk).

Los casos de agammaglobulinemia autosómica recesiva (ARA) también se observaron desde la época de Charles A Janeway; el conocimiento de la estructura del pre-BCR y la señalización del mismo facilitaron la determinación de los defectos genéticos en la agammaglobulinemia autosómica recesiva. A la fecha se conocen siete genes implicados en esta enfermedad; entre éstos están los genes que codifican para $\lg \mathrm{H} \mu$, $\lambda 5, \lg \alpha, \lg \beta$, BLNK, PI3Kp85 y LRRC8A, todos estas moléculas (con excepción de LRRC8A, de la que aún se desconoce su función) forman parte del pre-BCR o de su vía de señalización. ${ }^{2}$

\section{Síndrome de hiper-IgM}

Otro síndrome que se asocia con defectos funcionales en las células B es el síndrome de hiper-lgM, entidad clínica descrita a principios del decenio de 1960 por Charles A Janeway. ${ }^{8}$ Desde su descripción, el síndrome de hiper-IgM se consideró un defecto intrínseco de las células B; sin embargo, la descripción del defecto genético asociado con la forma ligada al cromosoma X (XHIGM) puso de manifiesto la importancia de la cooperación T-B. En 1985, Lloyd Mayer y Henry Kunkel, al realizar cocultivos de estas células T de linfoma con células B de pacientes con síndrome de hiper-lgM ligado al cromosoma $X$ descubrieron que estas últimas eran capaces de realizar el cambio de isotipo y expresar $\lg G$ e IgA. ${ }^{9}$ El defecto genético causante del síndrome de hiper-IgM ligado al cromosoma X lo describieron en 1993 cinco grupos de manera independiente, y resultó ser CD40L, molécula que justo un año antes se describió desde el punto de vista funcional; molécula expresada por los linfocitos $T^{23,24}$ y que ahora se sabe es decisiva para la inducción del cambio de isotipo en las células B. ${ }^{2}$

El descubrimiento de que CD40L es la molécula afectada en pacientes con el síndrome de hiperIgM dio la pauta para la generación de ratones deficientes de CD40, que muestran un fenotipo similar al del síndrome de hiper-lgM, porque esos ratones son incapaces de montar una respuesta humoral a antígenos timo-dependientes. ${ }^{25}$

En años posteriores se describieron los defectos genéticos asociados con el síndrome de hiper-IgM autosómico recesivo (AR-HIGM); el defecto genético en CD40 se describió en seres humanos en 2001, cuando se observó que las mutaciones en CD40 son responsables de un síndrome indistinguible desde el punto clínico de la forma de síndrome de hiper-IgM ligado al cromosoma $X^{2}{ }^{26}$ de manera adicional se han descrito mutaciones en los genes de ADA, UNG y proteínas de reparación de ADN (PMS2, LIG4, cerunnos, entre otros); la deficiencia genética de estos últimos no sólo da lugar a defectos en el cambio de isotipo en el ADN, sino que además se asocia con fragilidad cromosómica, generación de cáncer y facies características en los pacientes que la padecen. ${ }^{2}$

\section{Inmunodeficiencia común variable}

La inmunodeficiencia común variable fue el término usado por Charles A Janeway en 1953 para definir a los pacientes con hipogammaglobulinemia de IgG, IgA, IgM o las tres; ${ }^{10}$ este término lo usó posteriormente Max D Cooper, en 1978, en la primera clasificación de las inmunodeficiencias primarias, ${ }^{12}$ que se usa hasta la fecha. Los hallazgos clínicos asociados con este síndrome comprenden concentraciones bajas de dos de las tres inmunoglobulinas principales en suero (IgG, $\lg A$ e $\lg M)$, con existencia de linfocitos $\mathrm{B}$ en sangre periférica. ${ }^{27} \mathrm{La}$ manifestación clínica de esta enfermedad es muy 
variable: los pacientes pueden cursar con alteraciones gastrointestinales (enteropatía, enfermedad inflamatoria del intestino), autoinmunidad (con predominio de anemia hemolítica autoinmunitaria y trombocitopenia idiopática), formación de granuloma y linfomas, además de que se han reportado alteraciones funcionales en diversos tipos celulares, como linfocitos $T$, linfocitos $B$, células dendríticas, monocitos, entre otros. ${ }^{27}$ Asimismo, los defectos genéticos que se han identificado a la fecha también son variables.

La variabilidad en el fenotipo clínico de los pacientes con inmunodeficiencia común variable se ve reflejada en los genes asociados con esta enfermedad, porque, a diferencia de los genes asociados con agammaglobulinemia y síndrome de hiper-IgM, que están implicados en la señalización de pre-BCR y en el cambio de isotipo, respectivamente, los genes asociados con inmunodeficiencia común variable participan en diversas vías de señalización. ${ }^{28}$

A pesar de ser una enfermedad descrita desde 1953, la descripción del primer defecto genético asociado con inmunodeficiencia común variable se hizo en 2003, cuando Grimbacher y colaboradores describieron mutaciones homocigotas en el coestimulador inducible (ICOS), que es una molécula expresada por las células $T$ y que al interactuar con su ligando (ICOSL, expresado en las células presentadoras de antígeno), induce una respuesta de citocinas, como IL-4, IL-5 e IL10, que favorecen la polarización hacia Th2 y, en consecuencia, la producción de anticuerpos en los linfocitos B. ${ }^{29}$

El siguiente defecto genético que se describió, fue $\mathrm{TACl}$ (por sus siglas en inglés de transmembrane activator and (ALM interactor), un receptor expresado por los linfocitos $B$ que interactúa con el factor activador de la célula $B$ (BAFF) y el ligando inductor de la proliferación (APRIL). La interacción de TACI con sus ligandos (BAFF y APRIL) favorece la supervivencia, proli- feración y la producción de inmunoglobulinas por parte de los linfocitos B. ${ }^{30}$ La deficiencia de TACI se describió en un grupo de pacientes adultos con concentraciones disminuidas de linfocitos B de memoria con cambio de isotipo (CD19+IgD-CD27+), y que mostraban datos de linfoproliferación (hepatomegalia, esplenomegalia, adenomegalia, o autoinmunidad como tiroiditis, anemia perniciosa y vitíligo). ${ }^{31,32} \mathrm{La}$ mayoría de los pacientes con defectos en TACl los heredan en un patrón autosómico dominante; es decir, que basta con que uno de los alelos que codifican para $\mathrm{TACl}$ tenga la mutación para que se manifieste la inmunodeficiencia común variable; sin embargo, también existen casos autosómicos recesivos y heterocigotos compuestos, aunque con menor frecuencia; ${ }^{33}$ asimismo, se han reportado individuos sanos con los mismos cambios genéticos presentes en los pacientes con inmunodeficiencia común variable, por lo que aún queda por determinar si existen otros factores genéticos o ambientales que pudieran influir en la aparición de esta enfermedad. La deficiencia del receptor de BAFF (BAFFR) se describió en años posteriores; esta deficiencia se asocia con defectos en la generación de células $\mathrm{B}$ de memoria con cambio de isotipo, pero a diferencia de la deficiencia de $\mathrm{TACl}$, no existen síntomas de autoinmunidad. ${ }^{34}$

De manera adicional, se han descrito defectos genéticos que afectan al correceptor de la célula B formado por CD19, CD21 y CD81 en pacientes con inmunodeficiencia común variable. Mano van Zelm y colaboradores describieron en 2006 cinco pacientes provenientes de dos familias con mutaciones homocigotas en el gen que codifica para CD19; posteriormente se detectó un solo paciente con deficiencia de CD81 y otro con deficiencia de CD21; ${ }^{35-37}$ mientras que la deficiencia de CD19 y CD21 se correlaciona con disminución de células $\mathrm{B}$ de memoria con cambio de isotipo (CD19+lgM-CD27+) e hipogammaglobulinemia, la deficiencia de CD81 se manifestó, además, con autoinmunidad, 
posiblemente debido a que CD81 se expresa en diferentes tipos celulares, incluidos los timocitos, en los que podría modular la señalización del receptor de estas células (TCR). ${ }^{38}$

Otro grupo de defectos que puede identificarse en la inmunodeficiencia común variable son los que afectan a IL-21 y su receptor (IL-21R). La deficiencia de IL-21R se asocia con alteraciones en activación, formación de células $B$ de memoria y cambio de isotipo en los linfocitos B, además de defectos en la citotoxicidad mediada por las células NK y en la producción de diversas citocinas por parte de las células T. ${ }^{39}$ Asimismo, las mutaciones homocigotas en IL-21 inducen un fenotipo de inmunodeficiencia común variable asociado con enfermedad inflamatoria del intestino (IBD); sin embargo, aún no es claro cuál es el papel de IL-21/IL-21R en la patogénesis de la enfermedad inflamatoria del intestino; posiblemente se deba a deficiencias en la secreción de IL-10 causadas por la deficiencia de IL-21.40

La deficiencia de PLCy2 surgió recientemente como causa de inmunodeficiencia común variable; de manera similar a los defectos listados en inmunodeficiencia común variable, los pacientes padecen defectos en la generación de células $B$ de memoria con cambio de isotipo y en la producción de anticuerpos. Es interesante que uno de los hallazgos clínicos asociados con esta deficiencia es que los pacientes, además de autoinmunidad, tienen deficiencias funcionales en las células NK acompañadas de urticaria al frío, originadas principalmente por una degranulación espontánea de los mastocitos a temperaturas menores a $37^{\circ} \mathrm{C} .{ }^{41}$

Los defectos genéticos en PIK3CD, que codifica para la subunidad catalítica p110 de la fosfoinositido 3-cinasa (PI3K), se asocian con una ganancia de función en esta enzima, porque las células T de los pacientes tienen hiperacti- vación de Akt, acompañada de defectos en la generación de células B y T de memoria, así como deficiencias funcionales en los linfocitos T citotóxicos. ${ }^{42}$ Los individuos con esta deficiencia genética padecen hipogammaglobulinemia; sin embargo, también se han detectado pacientes con concentraciones elevadas de IgM y reducidas de IgG e IgA, linfoproliferación, susceptibilidad a infecciones por microorganismos piógenos, viremia por el virus de Epstein-Barr, entre otros hallazgos. ${ }^{42,43}$ La importancia de esta molécula en la biología del linfocito B es apoyada por dos reportes adicionales: el primero de ellos es la deficiencia genética de la subunidad reguladora p85-PI3K, que se asocia con un síndrome clínico de agammaglobulinemia ${ }^{44}$ $y$, por otra parte, eliminaciones en PIK3R1 que ocasionan la pérdida de la expresión de p85, p55 y p50, causando un fenotipo clínico similar al observado en los pacientes con deficiencia en PIK3CD. ${ }^{45}$

Recientemente, las mutaciónes heterocigotas en CTLA4 se describieron en un grupo de pacientes con inmunodeficiencia común variable acompañada de autoinmunidad. En estos pacientes ocurre una disminución progresiva con la edad de las células B, además de la falla en diferenciación a linfocitos $\mathrm{B}$ de memoria; por otra parte, también ocurren alteraciones funcionales en los linfocitos T, especialmente defectos en la generación de linfocitos $\mathrm{T}$ reguladores (Treg), posiblemente debido a la disminución en la inducción de FoxP3; de manera adicional, la deficiencia de CTLA-4 se acompaña de hiperactivación de los linfocitos $T$ cooperadores y $T$ citotóxicos, y se asocia con autoinmunidad. ${ }^{46,47}$

Por último, la deficiencia en LRBA (por sus siglas en inglés de lipopolysaccharide-responsive and beige-like anchor) se asoció con defectos en la supervivencia de las células $T$ y las células B, así como con autofagia exacerbada y defectos en la diferenciación de las células B a células 
de memoria. ${ }^{48}$ Esta deficiencia se reportó en pacientes con autoinmunidad y mala respuesta a vacunas sin disminución en las concentraciones plasmáticas de las inmunoglobulinas y en pacientes con autoinmunidad tipo IPEX; es decir, pacientes con deficiencia en la generación de células $\mathrm{T}$ reguladoras. ${ }^{49} \mathrm{~A}$ diferencia de los defectos genéticos mencionados en la inmunodeficiencia común variable, de los que se conocen las vías de señalización que participan, en el caso de la deficiencia de LRBA no se conoce su función. El conocimiento de la secuencia de LRBA nos da una pauta para realizar hipótesis acerca de ésta: por ejemplo, no parece que LRBA tenga ningún tipo de actividad enzimática $y$, debido a que tiene ciertos dominios estructurales, como $\mathrm{PH}, \mathrm{SH} 3, \mathrm{SH} 2, \mathrm{BEACH}$ y WD40, se predice que su función es la de una proteína de andamiaje, aunque la(s) vía(s) de señalización en la(s) que está implicada se desconoce; sin embargo, LRBA tiene motivos AKAP (A-kinase anchored protein) que facilitarían su interacción con las subunidades reguladoras de la proteína dependiente de AMPc (PKA-R), además de otros dominios que podrían facilitar su interacción con proteínas de autofagia..$^{50-52}$ Los defectos genéticos mencionados se listan en el Cuadro 1.

Por último, la inmunodeficiencia común variable, junto con la agammaglobulinemia, son las inmunodeficiencias primarias más comúnmente diagnosticadas en el mundo (después de la deficiencia de IgA, que no se considera clínicamente grave, motivo por el que omitimos su discusión). Asimismo, mientras que casi el $100 \%$ de los casos de agammaglobulinemia se asocian con los defectos genéticos mencionados, los defectos en inmunodeficiencia común variable no cubren más de $20 \%$ de los casos con este diagnóstico, por lo que diversos grupos de científicos en el mundo han centrado sus investigaciones en el estudio de esta inmunodeficiencia primaria.
Cuadro 1. Principales inmunodeficiencias primarias humorales y los genes identificados en las mismas

\begin{tabular}{|c|c|c|}
\hline $\begin{array}{l}\text { Proteína } \\
\text { deficiente }\end{array}$ & Fenotipo clínico & Patrón de herencia \\
\hline $\begin{array}{l}\text { Btk } \\
\operatorname{lgH} \mu \\
\text { BLNK } \\
\lambda 5 \\
\lg \alpha \\
\lg \beta \\
\text { PI3K p85 } \\
\text { LRRC8A }\end{array}$ & $\begin{array}{c}\text { Agammaglobuli- } \\
\text { nemia }\end{array}$ & $\begin{array}{l}\text { Ligado al cromosoma X } \\
\text { Autosómico recesivo } \\
\text { Autosómico recesivo } \\
\text { Autosómico recesivo } \\
\text { Autosómico recesivo } \\
\text { Autosómico recesivo } \\
\text { Autosómico recesivo } \\
\text { Autosómico recesivo }\end{array}$ \\
\hline $\begin{array}{l}\text { CD40L } \\
\text { AID } \\
\text { CD40 } \\
\text { UNG }\end{array}$ & $\begin{array}{l}\text { Síndrome de } \\
\text { hiper-lgM }\end{array}$ & $\begin{array}{l}\text { Ligado al cromosoma } \mathrm{X} \\
\text { Autosómico recesivo } \\
\text { Autosómico recesivo } \\
\text { Autosómico recesivo }\end{array}$ \\
\hline $\begin{array}{l}\text { ICOS } \\
\text { TACI } \\
\text { BAFFR } \\
\text { CD19 } \\
\text { CD21 } \\
\text { CD81 } \\
\text { IL-21 } \\
\text { IL-21R } \\
\text { PLC 2 } \\
\text { PI3K p1108 } \\
\text { CTLA4 } \\
\text { LRBA }\end{array}$ & $\begin{array}{l}\text { Inmunodefi- } \\
\text { ciencia común } \\
\text { variable }\end{array}$ & $\begin{array}{l}\text { Autosómico recesivo } \\
\text { Autosómico dominante } \\
\text { Autosómico recesivo } \\
\text { Autosómico recesivo } \\
\text { Autosómico recesivo } \\
\text { Autosómico recesivo } \\
\text { Autosómico recesivo } \\
\text { Autosómico recesivo } \\
\text { Autosómico recesivo } \\
\text { Autosómico dominante } \\
\text { Autosómico dominante } \\
\text { Autosómico recesivo }\end{array}$ \\
\hline
\end{tabular}

Inmunodeficiencias primarias humorales en México

El estudio de este grupo de defectos inició en nuestro país hace poco más de una década. El primer síndrome analizado fue precisamente la agammaglobulinemia ligada al cromosoma X; se estudió una cohorte de pacientes diagnosticados desde el punto de vista clínico en el Instituto Nacional de Pediatría (INP), SSa, y el Centro Médico Nacional La Raza, IMSS. Después de un estudio fenotípico y genético se determinó el tipo de mutaciones en esos pacientes; se encontraron 14 mutaciones, dos de ellas resultaron ser nuevas; es decir, que no existía ningún reporte del mismo cambio genético encontrado por nuestro grupo..$^{53} \mathrm{En}$ la actualidad, el número de pacientes con agammaglobulinemia ligada al cromosoma $\mathrm{X}$ en los 
que se busca su defecto molecular, es de 70 , todos provienen de diferentes centros de salud, principalmente, el INP-SSa, el Centro Médico Nacional La Raza, IMSS, y el Centro Médico Nacional del Noreste, IMSS.

El hallazgo de mutaciones nuevas en BTK nos lleva a realizar estudios adicionales para demostrar la importancia de estas mutaciones; por ejemplo, las dos nuevas mutantes reportadas en $2008^{53}$ nos llevaron a comprobar la deficiencia en la función de la proteína utilizando un sistema de células B de pollo, knock-out para Btk. ${ }^{54}$

El síndrome de hiper-IgM ligado al cromosoma X ha sido objetivo de otro de nuestros proyectos de investigación, aunque el número de casos diagnosticados en la clínica es limitado; al respecto, se ha reportado una serie de seis pacientes con mutaciones en el gen que codifica para $\mathrm{CD}^{4} \mathrm{~L}^{55}$ y actualmente se estudian tres pacientes más que probablemente tengan este defecto.

Otro de los intereses de nuestro grupo de investigación es el estudio de los defectos genéticos en la inmunodeficiencia común variable. $\mathrm{Al}$ respecto, se cuenta con una cohorte de 70 pacientes con este diagnóstico, provenientes de diferentes centros de salud, como Centro Médico Nacional La Raza, IMSS, Centro Médico Nacional Siglo XXI, IMSS, y el Instituto Nacional de Pediatría, SSa. En estos pacientes pretendemos determinar si tienen alteraciones en el desarrollo periférico de los linfocitos B, identificando mediante la detección de marcadores de superficie, diferentes tipos celulares, como células B vírgenes, células B transicionales, células $B$ de memoria con y sin cambio de isotipo y células B con baja expresión de

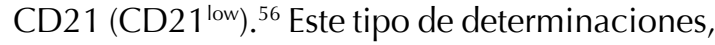
aunque no orienta el diagnóstico genético, es confirmatorio de inmunodeficiencia común variable y en algunos casos orienta el pronóstico de la misma, porque los individuos con un alto porcentaje de células B CD2 $1^{\text {low }}$ se asocian con la ocurrencia de enfermedades autoinmunitarias.

En la actualidad uno de nuestros intereses en el estudio de inmunodeficiencia común variable consiste en determinar la deficiencia de LRBA en pacientes mexicanos con este diagnóstico. Resulta interesante detectar individuos con mutaciones en este gen, para posteriormente profundizar en el estudio de la posible función de esta molécula en los linfocitos B, por ejemplo, la vía de señalización en la que esta molécula está implicada y las consecuencias funcionales que conlleva su deficiencia.

\section{CONCLUSIONES}

La descripción de las inmunodeficiencias primarias de anticuerpos y de los defectos genéticos con los que se asocian ha dependido, en gran medida, de los hallazgos científicos que se derivan acerca de las células B, básicamente de las vías de señalización que favorecen su diferenciación, supervivencia y activación. Asimismo, la descripción de los defectos genéticos causantes de este tipo de deficiencias jamás habría sido posible sin los grandes descubrimientos que involucran la descripción de la estructura del ADN, el código genético, la secuenciación del genoma humano, así como el surgimiento de los métodos de secuenciación, la reacción en cadena de la polimerasa y, recientemente, la secuenciación masiva del ADN.

En la actualidad vivimos una explosión de deficiencias genéticas que causan una inmunodeficiencia primaria; vemos cómo cada individuo con este tipo de diagnóstico podría ser único en el mundo; tal es el caso reportado para las deficiencias IL21/IL21-R y BAFFR. EI estudio de estas enfermedades ha contribuido, además, a identificar o ampliar el conocimien- 
to de los mecanismos moleculares que tienen lugar durante la activación de las células del sistema inmunológico. El entendimiento de estos mecanismos facilitará, en un momento determinado, el diagnóstico de nuevos casos y el tratamiento de los mismos.

\section{Agradecimientos}

Agradezco infinitamente a mi mentor, el Dr. Leopoldo Santos Argumedo, por incluirme en la participación del simposio para celebrar el 50 aniversario del descubrimiento del linfocito B. A los organizadores del evento (Sociedad Mexicana de Inmunología y al Departamento de Biología de la Facultad de Química, UNAM) y finalmente al CONACYT por los recursos que se me otorgaron para la realización de algunos de los proyectos que se mencionaron en este artículo.

\section{REFERENCIAS}

1. Casanova JL, Abel L. Primary immunodeficiencies: a field in its infancy. Science 2007;317:617-619.

2. Durandy A, Kracker S, Fischer A. Primary antibody deficiencies. Nature Reviews Immunology 2013;13:519-533.

3. Glanzmann E, Riniker P. [Essential lymphocytophthisis; new clinical aspect of infant pathology]. Annales Paediatrici International Review Pediatrics 1950;175:1-32.

4. Greenwood B. The contribution of vaccination to global health: past, present and future. Philosophical transactions of the Royal Society of London Series B. Biological Sciences 2014;369:20130433.

5. Bruton OC. Agammaglobulinemia. Pediatrics 1952;9:722728.

6. Bruton OC, Apt L, Gitlin D, Janeway CA. Absence of serum gamma globulins. AMA American J Dis Children 1952;84:632-636.

7. Ochs HD, Hitzig WH. History of primary immunodeficiency diseases. Current Op Allergy Clin Immunol 2012;12:577587.

8. Rosen FS, Kevy SV, Merler E, Janeway CA, Gitlin D. Recurrent bacterial infections and dysgamma-globulinemia: deficiency of 7S gamma-globulins in the presence of elevated $19 S$ gamma-globulins. Report of two cases. Pediatrics 1961;28:182-195.

9. Mayer L, Kwan SP, Thompson C, Ko HS, et al. Evidence for a defect in "switch" $T$ cells in patients with immunodefi- ciency and hyperimmunoglobulinemia M. N Engl J Med 1986;314:409-413.

10. Janeway CA, Apt L, Gitlin D. Agammaglobulinemia. Transactions of the Association of American Physicians 1953;66:200-202

11. Gitlin D, Janeway CA. Agammaglobulinemia, congenital, acquired and transient forms. Progress Hematol 1956;1:318-329.

12. Cooper MD, Faulk WP, Fudenberg HH, Good RA, et al. Classification of primary immunodeficiencies. N Engl J Med 1973;288:966-967.

13. Crabbe PA, Heremans JF. Lack of gamma A-immunoglobulin in serum of patients with steatorrhoea. Gut 1966;7:119127.

14. Schur PH, Borel H, Gelfand EW, Alper CA, Rosen FS. Selective gamma-g globulin deficiencies in patients with recurrent pyogenic infections. N Engl J Med 1970;283:631-634.

15. Umetsu DT, Ambrosino DM, Quinti I, Siber GR, Geha RS. Recurrent sinopulmonary infection and impaired antibody response to bacterial capsular polysaccharide antigen in children with selective IgG-subclass deficiency. N Engl J Med 1985;313:1247-1251.

16. Smith $\mathrm{Cl}$, Islam KB, Vorechovsky I, Olerup O, et al. X-linked agammaglobulinemia and other immunoglobulin deficiencies. Immunol Rev 1994;138:159-183.

17. Giblett ER. ADA and PNP deficiencies: how it all began. Ann NY Acad Sci 1985;451:1-8.

18. Raff MC, Megson M, Owen JJ, Cooper MD. Early production of intracellular IgM by B-lymphocyte precursors in mouse. Nature 1976;259:224-226.

19. Sakaguchi N, Melchers F. Lambda 5, a new light-chainrelated locus selectively expressed in pre-B lymphocytes. Nature 1986;324:579-582.

20. Kudo A, Melchers F. A second gene, VpreB in the lambda 5 locus of the mouse, which appears to be selectively expressed in pre-B lymphocytes. EMBO J 1987;6:2267-2272.

21. Tsukada S, Saffran DC, Rawlings DJ, Parolini O, et al. Deficient expression of a B cell cytoplasmic tyrosine kinase in human X-linked agammaglobulinemia. Cell 1993;72:279290.

22. Vetrie D, Vorechovsky I, Sideras P, Holland J, et al. The gene involved in X-linked agammaglobulinaemia is a member of the src family of protein-tyrosine kinases. Nature 1993;361:226-233.

23. Spriggs MK, Armitage RJ, Strockbine L, Clifford KN, Macduff $B M$, et al. Recombinant human CD40 ligand stimulates $B$ cell proliferation and immunoglobulin E secretion. J Exp Med 1992;176:1543-1550.

24. Lederman S, Yellin MJ, Inghirami G, Lee JJ, et al. Molecular interactions mediating T-B lymphocyte collaboration in human lymphoid follicles. Roles of T cell-B-cell-activating molecule (5c8 antigen) and CD40 in contact-dependent help. J Immunol 1992;149:3817-3826. 
25. Renshaw BR, Fanslow WC, 3rd, Armitage RJ, Campbell KA, et al. Humoral immune responses in CD40 ligand-deficient mice. J Exp Med 1994;180:1889-1900.

26. Ferrari S, Giliani S, Insalaco A, Al-Ghonaium A, et al. Mutations of CD40 gene cause an autosomal recessive form of immunodeficiency with hyper IgM. Proc Natl Acad Sci USA 2001;98:12614-12619.

27. Yong PF, Thaventhiran JE, Grimbacher B. "A rose is a rose is a rose", but CVID is not CVID common variable immune deficiency (CVID), what do we know in 2011? Advances Immunol 2011;111:47-107.

28. Jolles $\mathrm{S}$. The variable in common variable immunodeficiency: a disease of complex phenotypes. J Allergy Clinical Immunol Practice 2013;1:545-556; quiz 557.

29. Grimbacher B, Hutloff A, Schlesier M, Glocker E, et al. Homozygous loss of ICOS is associated with adultonset common variable immunodeficiency. Nat Immunol 2003;4:261-268.

30. Vincent FB, Saulep-Easton D, Figgett WA, Fairfax KA, Mackay F. The BAFF/APRIL system: emerging functions beyond $B$ cell biology and autoimmunity. Cytokine Growth Factor Rev 2013;24:203-215.

31. Castigli E, Wilson SA, Garibyan L, Rachid R, et al. TACl is mutant in common variable immunodeficiency and IgA deficiency. Nat Genet 2005;37:829-834.

32. Salzer U, Chapel HM, Webster AD, Pan-Hammarstrom Q, et al. Mutations in TNFRSF13B encoding TACl are associated with common variable immunodeficiency in humans. Nat Genet 2005;37:820-828.

33. Salzer U, Bacchelli C, Buckridge S, Pan-Hammarstrom Q, et al. Relevance of biallelic versus monoallelic TNFRSF13B mutations in distinguishing disease-causing from riskincreasing TNFRSF13B variants in antibody deficiency syndromes. Blood 2009;113:1967-1976.

34. Warnatz K, Salzer U, Rizzi M, Fischer B, et al. B-cell activating factor receptor deficiency is associated with an adult-onset antibody deficiency syndrome in humans. Proc Natl Acad Sci USA 2009;106:13945-13950.

35. van Zelm MC, Reisli I, van der Burg M, Castano D, et al. An antibody-deficiency syndrome due to mutations in the CD19 gene. N Engl J Med 2006;354:1901-1912.

36. van Zelm MC, Smet J, Adams B, Mascart F, et al. CD81 gene defect in humans disrupts CD19 complex formation and leads to antibody deficiency. J Clin Invest 2010;120:1265-1274.

37. Thiel J, Kimmig L, Salzer U, Grudzien M, et al. Genetic CD21 deficiency is associated with hypogammaglobulinemia. J Allergy Clin Immunol 2012;129:801-810.

38. Cevik SI, Keskin N, Belkaya S, Ozlu MI, et al. CD81 interacts with the $\mathrm{T}$ cell receptor to suppress signaling. PloS one. 2012;7:e50396.

39. Kotlarz D, Zietara N, Uzel G, Weidemann T, et al. Lossof-function mutations in the IL-21 receptor gene cause a primary immunodeficiency syndrome. J Exp Med 2013;210:433-443.
40. Doganci A, Birkholz J, Gehring S, Puhl AG, et al. In the presence of IL-21 human cord blood T cells differentiate to IL-10-producing Th1 but not Th17 or Th2 cells. Int Immunol 2013;25:157-169.

41. Ombrello MJ, Remmers EF, Sun G, Freeman AF, et al. Cold urticaria, immunodeficiency, and autoimmunity related to PLCG2 deletions. N Engl J Med 2012;366:330-338.

42. Lucas CL, Kuehn HS, Zhao F, Niemela JE, et al. Dominantactivating germline mutations in the gene encoding the $\mathrm{PI}(3) \mathrm{K}$ catalytic subunit $\mathrm{p} 110$ delta result in $\mathrm{T}$ cell senescence and human immunodeficiency. Nat Immunol 2014;15:88-97.

43. Crank MC, Grossman JK, Moir S, Pittaluga S, et al. Mutations in PIK3CD can cause hyper IgM syndrome (HIGM) associated with increased cancer susceptibility. J Clin Immunol.2014;34:272-276.

44. Conley ME, Dobbs AK, Quintana AM, Bosompem A, et al. Agammaglobulinemia and absent $B$ lineage cells in a patient lacking the p85alpha subunit of PI3K. J Exp Med 2012;209:463-470.

45. Lucas $\mathrm{CL}$, Zhang $\mathrm{Y}$, Venida $\mathrm{A}$, Wang $\mathrm{Y}$, et al. Heterozygous splice mutation in PIK3R1 causes human immunodeficiency with lymphoproliferation due to dominant activation of PI3K. J Exp Med 2014;211:2537-2547.

46. Kuehn HS, Ouyang W, Lo B, Deenick EK, et al. Immune dysregulation in human subjects with heterozygous germline mutations in CTLA4. Science 2014;345:1623-1627.

47. Schubert D, Bode C, Kenefeck R, Hou TZ, et al. Autosomal dominant immune dysregulation syndrome in humans with CTLA4 mutations. Nature Med 2014;20:1410-1416.

48. Lopez-Herrera G, Tampella G, Pan-Hammarstrom Q, Herholz $\mathrm{P}$, et al. Deleterious mutations in LRBA are associated with a syndrome of immune deficiency and autoimmunity. Am J Human Gen 2012;90:986-1001.

49. Charbonnier LM, Janssen E, Chou J, Ohsumi TK, et al. Regulatory T-cell deficiency and immune dysregulation, polyendocrinopathy, enteropathy, X-linked-like disorder caused by loss-of-function mutations in LRBA. J Allergy Clin Immunol 2015;135:217-227.

50. Wang JW, Howson J, Haller E, Kerr WG. Identification of a novel lipopolysaccharide-inducible gene with key features of both A kinase anchor proteins and chs1/beige proteins. J Immunol 2001;166:4586-4595.

51. Wang JW, Gamsby JJ, Highfill SL, Mora LB, et al. Deregulated expression of LRBA facilitates cancer cell growth. Oncogene 2004;23:4089-4097.

52. Cullinane $A R$, Schaffer $A A$, Huizing $M$. The BEACH is hot: a LYST of emerging roles for BEACH-domain containing proteins in human disease. Traffic 2013;14:749-766.

53. Lopez-Herrera G, Berron-Ruiz L, Mogica-Martinez D, Espinosa-Rosales F, Santos-Argumedo L. Characterization of Bruton's tyrosine kinase mutations in Mexican patients with X-linked agammaglobulinemia. Molecular Immunol 2008;45:1094-1098. 
54. Vargas-Hernandez A, Lopez-Herrera G, Maravillas-Montero $\mathrm{JL}$, Vences-Catalan $\mathrm{F}$, et al. Consequences of two naturally occurring missense mutations in the structure and function of Bruton agammaglobulinemia tyrosine kinase. IUBMB life 2012;64:346-353.

55. Vargas-Hernandez A, Berron-Ruiz L, Staines-Boone T, Zarate-Hernandez $M$, et al. Clinical and genetic analysis of patients with X-linked hyper-IgM syndrome. Clinical Gen 2013;83:585-587.
56. Berron-Ruiz L, Lopez-Herrera G, Vargas-Hernandez A, Mogica-Martinez D, et al. Lymphocytes and B-cell abnormalities in patients with common variable immunodeficiency (CVID). Allergol Immunopathol 2014;42:35-43.

\section{Fuentes electrónicas}

http://esid.org/Working-Parties/Registry/ESID-DatabaseStatistics. 